\title{
Author Correction: Electrotunable nanoplasmonic liquid mirror
}

Yunuen Montelongo, Debabrata Sikdar, Ye Ma, Alastair J. S. McIntosh, Leonora Velleman, Anthony R. Kucernak, Joshua B. Edel and Alexei A. Kornyshev

Correction to: Nature Materials https://doi.org/10.1038/nmat4969, published online 11 September 2017.

In the version of this Article originally published, the last sentence of the acknowledgements incorrectly read 'L.V. acknowledges the support of a Marie Skodowska-Curie fellowship (N-SHEAD)'; it should have read 'L.V. and D.S. acknowledge the support of Marie Skłodowska-Curie fellowships, N-SHEAD and S-OMMs, respectively'.

Published online: 10 May 2019

https://doi.org/10.1038/s41563-019-0392-6 